\title{
Vinculando la enseñanza de la independencia nacional con temas socialmente relevantes para la formación de ciudadanos"
}

Connecting the teaching of the National Independence

with socially relevant issues for the formation of citizens

\author{
Gabriel Villalón-GálveZ ${ }^{a}$, Eduardo CaVieres-Fernández ${ }^{b}$ \& Gabriela VÁsQuez-Leyton ${ }^{c}$ \\ aUniversidad de Chile, Santiago, Chile. $\nabla$ gabriel.villalon@uchile.cl \\ [orcid.org/0000-0003-0580-1870] \\ bUniversidad de Playa Ancha. ecavieres-cea@upla.cl \\ [orcid.org/0000-0003-3199-5090] \\ cUniversidad Andrés Bello.gabriela.vasquez@unab.cl \\ [orcid.org/0000-0002-5633-7572]
}

\section{RESUMEN}

El objetivo de este trabajo es analizar, a través de una aproximación exploratoria cualitativa, y desde la perspectiva de una profesora de Historia de la ciudad de Arica, la vinculación que establece entre la enseñanza de la Historia, los contenidos de enseñanza sobre la Independencia Nacional y las temáticas relevantes para la formación ciudadana de sus estudiantes. A partir de los hallazgos, discutimos la pertinencia de determinados modos de enseñar la Historia en vistas a establecer vinculaciones con la realidad de los estudiantes, y cómo ello ayuda a vincular contenidos históricos más distantes en el tiempo con temáticas socialmente relevantes que aportan a su formación ciudadana.

PALABRAS CLAVE: Profesores de Historia, Enseñanza de la Historia, Enseñanza de la Independencia Nacional, Temas Socialmente Relevantes, Educación Ciudadana.

\section{ABSTRACT}

The goal of this study is to analyze, from the perspective of a History teacher from the city of Arica, the connection that she establishes between History teaching, the teaching contents on the National Independence and relevant issues for the citizenship education of her students. Based on our findings, we discuss the relevance of particular ways of teaching

Esta investigación ha sido realizada gracias al financiamiento de FONDECYT (Fondo Nacional de Desarrollo Científico y Tecnológico) Proyecto $\mathrm{N}^{\circ} 1180475$. 
History for establishing connections with the students, and how this helps to connect historical contents that are more distant in time with socially relevant issues that contribute to their citizenship formation.

KEY WORDS: History Teachers, History Teaching, Teaching of the National Independence; Socially Relevant Issues, Citizenship Education.

\section{INTRODUCCIÓN}

Los avances en la enseñanza de la historia escolar, han traído un cambio paradigmático en los modos de establecer objetivos y metodologías en este ámbito curricular (Metzger \& Harris, 2018). Con ello, se ha pasado de un modelo centrado en la promoción de narrativas sobre la construcción del Estado-Nación, en torno a las cuales debían articularse las identidades ciudadanas, a otro que pone primeramente su acento en la inserción de los ciudadanos en una sociedad plural (Nordgren \& Johansson, 2015; Pagès, Villalón \& Zamorano, 2017). Ello, a su vez, ha exigido fomentar en los estudiantes el pensamiento histórico, permitiéndoles comprender los procesos de cambio/continuidad en el tiempo y su influencia en la sociedad del presente, y, consiguientemente, proveyéndoles de las habilidades reflexivas requeridas para problematizar críticamente el pasado, tal como ha sido transmitido, y así generar vinculación con aquellos procesos y eventos que dan sentido y explican los acontecimientos del hoy en vistas a los desafíos ciudadanos del futuro (Seixas, 2017; Barton \& Levstik, 2009).

A la luz de lo anterior, resulta una tarea fundamental analizar la pertinencia entre determinados contenidos históricos y la formación ciudadana de los estudiantes. Ello implica que dichos contenidos no sean meras descripciones de eventos del pasado, sino que permitan discutir sus supuestos e implicancias desde las distintas perspectivas que aún condicionan los debates en la sociedad. Ante ello, mientras algunos autores los consideran controversiales (Barton \& Mc Cully, 2007), en cuanto generan posturas contrarias que permiten su discusión; otros proponen trabajarlos como temáticas públicas socialmente relevantes, pues suponen asuntos conflictivos en la sociedad, tanto del pasado como actuales, que deben ser reflexionados críticamente (Goldberg \& Savenije, 2018; Santisteban, 2019). No obstante, la tendencia ha sido proponer contenidos vinculados a hechos de la historia reciente, dada la polarización que generan en la actualidad, y, por tanto, más propicios para generar discusión. Aquello tiende a relegar eventos de más larga data en el tiempo, con menor impacto en la sensibilidad de los estudiantes, pero que, en vistas a la formación de su conciencia histórica crítica y ciudadana, podrían permitirles, a través de su problematización, comprender y discutir los conflictos que suscitaron en el pasado y relacionarlos sobre sus efectos que aún perduran en el presente. Dado el innegable valor histórico de la Independencia Nacional, en principio, esta temática podría logra dicha intencionalidad (Pinto \& Valdivia, 2009).

Por lo tanto, usando una aproximación exploratoria que se justifica dada la escasez relativa de trabajos en este ámbito de investigación (Neuman, 2014), el objetivo de este trabajo es analizar, desde la perspectiva de una profesora de la ciudad de Arica, la relación que establece entre la enseñanza de la historia, los contenidos sobre la Independencia Nacional 
y temáticas relevantes para la formación ciudadana de sus estudiantes. Consiguientemente, a continuación, presentamos nuestro marco conceptual sustentado en la literatura acerca de la relación entre la enseñanza de la historia y su relación con la formación ciudadana, y el rol que cumplen los profesores al promover el tratamiento de temas socialmente relevantes en el aula. Luego, teniendo presente dicho marco conceptual y a partir de los hallazgos, discutimos el uso, por parte de la profesora, de una metodología no lineal de la enseñanza de la historia para establecer vinculaciones con la realidad de sus estudiantes; la que incluyó establecer relaciones entre la temática de la Independencia Nacional y temáticas socialmente relevantes que aportan a la formación ciudadana de estos estudiantes. También ofrecemos algunas líneas de investigación que requieren ser profundizadas en el futuro considerando las conclusiones preliminares de este estudio.

\section{MARCO CONCEPTUAL}

\section{Enseñanza de la historia y formación ciudadana}

Un primer sustento teórico de este trabajo viene dado por las nuevas comprensiones acerca de cómo enseñar la historia escolar. Estas comprensiones se sitúan críticamente frente a la tradicional relación establecida entre el Estado-Nación y la escuela para imponer narrativas oficiales que generen en la población consenso y adhesión social (Carretero, Asensio \& Rodríguez-Moneo, 2012; Pagès, 2016). Consecuentemente, la asignatura de la Historia ha servido para transmitir a las nuevas generaciones, a través del currículo, interpretaciones sobre eventos, símbolos y personajes del pasado, afines a determinados grupos dominantes, legitimando y perpetuando nociones acerca de cómo debe construirse la sociedad (Rosa \& Brescó, 2017). Ello, a su vez, ha supuesto un modelo pasivo de formación ciudadana, conforme al cual, la participación se reduce a colaborar en la institucionalidad política y legal de acuerdo a los procedimientos establecidos por el Estado. En la práctica, ello ha implicado que la vinculación entre historia escolar y ciudadanía quede poco explicitada y, más bien, sea sustituida por un modo de aprender la historia que es lineal y basada, fundamentalmente, en la asimilación de datos a través de los cuales se transmite una versión progresiva y romántica del Estado-Nación (Carretero, 2007).

Por contraste, diversos autores plantean la necesidad de promover nuevos modos de enseñar la historia que estén intrínsecamente unidos con una formación ciudadana que prepare a los estudiantes con los conocimientos y habilidades requeridas para participar activamente en los asuntos públicos (Arthur, Davies, Wrenn, Haydn \& Kerr, 2001). En esta perspectiva, la enseñanza de la historia ayuda a los estudiantes a desarrollar pensamiento histórico, a través del cual, acceden a conocimientos del pasado con el fin de entender mejor las problemáticas del presente y los desafíos del futuro que caracterizan a sus sociedades. Para ello, además, se insiste en el carácter interpretativo del conocimiento histórico -transmitido por medio de narrativas que, en sí mismas, son parciales y selectivas- lo que ayuda a comprender mejor los procesos de conformación de las instituciones políticas y culturales, los que han sido conflictivos y excluyentes (Haste \& Bermúdez, 2017). Consecuentemente, el pensamiento 
histórico contribuye a la formación de una conciencia histórica que permite a los estudiantes problematizar la vida social y, por tanto, les prepara para la convivencia en una sociedad que es compleja y necesita de constantes transformaciones si ha de ser más inclusiva (Seixas, 2017).

Lo anterior, por tanto, requiere de la formación de un conjunto de habilidades de pensamiento histórico en los estudiantes. En lo fundamental, se les debe ayudar a reflexionar críticamente para que establezcan relaciones causales entre procesos y eventos de la historia, realizar comparaciones entre períodos históricos para visualizar similitudes y diferencias, reconocer procesos de continuidad y cambio histórico, y contextualizar los hechos del pasado para posibilitar una mejor comprensión de ellos (van Boxtel y van Drie, 2018). Asimismo, aquello exige fomentar en los estudiantes habilidades argumentativas para que, reconociendo las múltiples interpretaciones que aporta el conocimiento histórico, analicen las narrativas heredadas, y formulen sus propias interpretaciones respecto de los hechos históricos (Carpente \& López Facal, 2013). Esto, a su vez, tiene implicancias para formar, en los estudiantes, la capacidad para vincular los procesos históricos del pasado con problemas actuales y deliberar soluciones en vistas al futuro de la sociedad. Ello resalta el valor público del aprendizaje de la Historia, la que posibilita la construcción de espacios en donde la discusión, en torno a temáticas sociales vigentes a lo largo del tiempo, es decisiva para la vida ciudadana y democrática de los estudiantes (De Amézola, 2008).

\section{Contenidos y temáticas socialmente relevantes}

Un aspecto fundamental para generar la vinculación entre la enseñanza de la Historia y la Formación Ciudadana se relaciona con los contenidos de enseñanza propiamente tales. Desde esa perspectiva, estos contenidos deben cumplir con el doble objetivo de relacionar hechos del pasado con problemáticas del presente y posibilitar perspectivas críticas que promuevan tanto el desarrollo del pensamiento histórico como habilidades para participar activamente en la sociedad. Ello significa que los contenidos a ser trabajados por los estudiantes digan relación con los efectos o consecuencias que provienen del pasado y continúan siendo influyentes en el modo cómo los ciudadanos se relacionan en la sociedad actual (Pagès, 2019). En ese contexto, una aproximación particularmente pertinente para generar pensamiento crítico en los estudiantes, es enfrentarlos a contenidos referidos a situaciones o eventos, originados a partir del conflicto de intereses o de valores entre grupos, y que han tenido un carácter perenne en la sociedad (McAvoy \& Hess, 2013). De este modo, estos contenidos cumplen un rol informativo al contextualizar históricamente los conflictos sociales ayudando a entender sus causas y alcances; y, por tanto, contribuyendo con perspectivas para deliberar sobre sus implicancias actuales (López Facal \& Santidrián, 2011).

Diversos autores conceptualizan dichos contenidos como temáticas controversiales o como problemas sociales relevantes (Swalwell \& Schweber, 2016; Ocampo \& Valencia, 2019). Estos contenidos, al sustentarse sobre conflictos a nivel social, integran interpretaciones diversas, frente a las cuales, se establecen posturas contrapuestas que reflejan las divisiones presentes en la sociedad más amplia (Schuitema, Radstake, van de Pol \& Veugelers, 2018). Consecuentemente, un objetivo asociado a ellos, es favorecer que los estudiantes los analicen 
críticamente a fin de establecer conclusiones propias respecto de los valores en juego, y sugerir alternativas para resolver los antagonismos presentes en la sociedad (Magendzo, 2016). En algunos casos, esto implica ampliar el alcance de las perspectivas propuestas en el currículo sobre determinados acontecimientos, ligado a versiones oficiales, e incorporar otras interpretaciones que igualmente circulan en la sociedad y que cuestionan dichas narrativas oficiales (Goldberg \& Savenije, 2018). De igual modo, suponen metodologías de enseñanza que favorezcan el protagonismo de los estudiantes para consultar fuentes diversas y reflexionar, individual y grupalmente, sobre estas temáticas, con el fin de discutir las diferentes posiciones en conflicto (Pineda, 2015).

Si bien, en principio, cualquier hecho histórico puede ser vinculado a problemáticas actuales y viceversa, diversas investigaciones proponen contenidos de enseñanza específicos dados sus efectos que aún repercuten en la sociedad y, cuyo análisis, por tanto, es particularmente pertinente a la formación ciudadana de los estudiantes. Consecuentemente, varios autores plantean la conveniencia de enseñar ciertos acontecimientos bélicos o periodos violentos del pasado que aún generan polarización en sus países (Zembylas \& Kambani, 2012; Kello, 2016); mientras algunos proponen temáticas más distantes en el tiempo, pero que afectan fenómenos aún presentes como la construcción de la identidad nacional (Sáiz \& López Facal, 2012). En Chile, entre los escasos estudios que analizan la enseñanza de hechos históricos en conexión con aspectos controversiales, figuran aquellos focalizados en eventos de la historia reciente (Salinas \& Oller, 2017; Vásquez, Sánchez, Vásquez \& Muñoz, 2018), con una ausencia generalizada de investigaciones centradas en eventos más alejados en el tiempo como lo sería la Independencia Nacional. No obstante, el trabajo de Miralles, Prats y Tatjer (2012), en el contexto español, resalta los aportes que el estudio de las Independencias Nacionales Iberoamericanas puede hacer a la reflexión que los estudiantes realizan en torno a temas multiculturales. Este estudio, se construye sobre estos hallazgos.

\section{Profesores y la enseñanza de la Historia desde una perspectiva socialmente relevante}

Un último componente teórico que sustenta esta investigación se refiere a la centralidad de los profesores en vincular la enseñanza de la Historia con temáticas socialmente relevantes. Al respecto, varios estudios subrayan el papel de los profesores en seleccionar metodologías que favorezcan un clima apropiado para trabajar temáticas controversiales en el aula (Hess, 2011). Ello incluye determinar las tareas y preguntas que debieran guiar a los estudiantes a niveles más altos de reflexión y discusión. Asimismo, los profesores aportan referentes contextuales que ayudan a entender las problemáticas analizadas, y mueven a los estudiantes para que expresen sus diferentes perspectivas, influidas por sus propios contextos culturales (Barton \& McCully, 2007). Finalmente, cumplen una labor seleccionando las temáticas a ser deliberadas, las que debieran ser relevantes en el ámbito público a fin de asegurar su pertinencia a la formación ciudadana de los estudiantes (Hess \& McAvoy, 2015). En el conjunto de estas tareas, el propio posicionamiento de los profesores frente a estos temas es fundamental ya que contribuye a generar un clima de discusión sin caer en adoctrinamientos o en la exclusión de los estudiantes (Conrad, 2020). 
Asimismo, una perspectiva de investigación importante considerada en este estudio ahonda en las comprensiones de los profesores respecto de la ciudadanía y su interrelación con aquellas referidas a la enseñanza de eventos históricos considerados controversiales (Niens, Connor \& Smith, 2013). Del mismo modo, Martínez-Rodríguez, Muñoz y SánchezAgustí (2019) advierten sobre las discordancias que pueden darse entre las concepciones críticas de los profesores sobre un hecho histórico reciente y sus formas de enseñarlo que no provocan reflexión crítica entre los estudiantes. En Chile, son escasos los estudios que abordan cuestiones similares, destacándose los trabajos de Toledo, Magendzo, Gutiérrez e Iglesias (2015), con profesores en ejercicio, y de Montanares-Vargas (2017), con futuros profesores en formación. En ellos se concluye que, tanto hechos de la Historia reciente como más distantes en el tiempo ocurridos en Chile, tienen un componente controversial, dada la violencia y división que resultaron de ellos. No obstante, por lo mismo, son difíciles de enseñar como controversiales por el conflicto que podrían generar en el aula y porque éste aspecto no está debidamente tratado en el currículo prescrito. En el presente trabajo se sigue explorando esta temática a partir de cómo una profesora vincula un hecho histórico con problemáticas actuales, lo que favorece la formación ciudadana de sus estudiantes.

\section{METODOLOGÍA}

En el presente trabajo, utilizamos una aproximación exploratoria cualitativa que se justifica dada la relativa escasez de trabajos en este ámbito de investigación (Neuman, 2014). Para ello se usan instrumentos cualitativos con el objetivo de analizar desde la perspectiva de una profesora de la ciudad de Arica, la relación que establece entre la enseñanza de la Historia, los contenidos sobre la Independencia Nacional y temáticas relevantes para la formación ciudadana de sus estudiantes

\section{Participantes}

En este estudio, distinguimos entre participantes principales y secundarios. La participante principal es una profesora de Historia de la ciudad de Arica, quien, al momento de esta investigación, llevaba 5 años de ejercicio docente y trabajaba en un establecimiento público. Junto a ello, tenía la responsabilidad de enseñar la unidad de la Independencia Nacional en octavo básico. Para su selección, se consultó a un programa de formación docente en la región sobre docentes que destacaban en el ámbito de la enseñanza de la Historia escolar. Se nos recomendó a esta profesora dado los servicios pedagógicos que prestaba en dicha unidad académica, así como por su sobresaliente preparación docente y desarrollo profesional. En este estudio es identificada con el seudónimo Sonia para mantener su anonimato. También se nos recomendó a los tres participantes secundarios (dos hombres y una mujer), con más de diez años de ejercicio docente, y que trabajaban en establecimientos municipales de la ciudad de Arica. 


\section{Recolección de datos}

Para la recolección de datos se utilizaron diversos instrumentos a fin de desarrollar el objetivo de investigación. En primer lugar, con la participante principal, se realizaron dos entrevistas individuales, de 45 minutos cada una; la primera de ellas a mediados de octubre del 2018 y la segunda a mediados de enero del 2019. En estas entrevistas, se consultó a la profesora sobre las temáticas que componen esta investigación, tales como la enseñanza de la Historia, la Formación Ciudadana y la Independencia Nacional como unidad de enseñanza; ahondando en la experiencia docente que la profesora tenía en estos temas. Adicionalmente, en cada entrevista, se profundizó en los datos recogidos por dos instrumentos adicionales cuya función era precisamente aportar elementos contextuales a ser considerados durante estas entrevistas.

El primer instrumento adicional utilizado fue la observación de clases. A mediados de octubre 2018, se observaron las dos primeras lecciones sobre la Independencia Nacional, tal cómo son propuestas por el texto escolar de Historia, Geografía y Ciencias Sociales para octavo básico distribuido por el Ministerio de Educación (Landa \& Pinto, 2017). Estas lecciones incluyen contenidos tales como la Ilustración y la Revolución Francesa, que anteceden aquellos referidos al proceso independista en América y en Chile. Las observaciones fueron registradas con grabadoras de audio y fueron transcritas en su totalidad. Adicionalmente, se tomaron notas de campo que sirvieron para elaborar preguntas que fueron incluidas en la primera entrevista con la profesora respecto de la dinámica que se dio en el aula; las conversaciones e interacciones que sostuvo con los estudiantes; los recursos que utilizó en su enseñanza, etc.

Como segundo instrumento adicional, a mediados de enero 2019, se realizó una entrevista grupal/focus group, con una duración de una hora, con la participante principal y los participantes secundarios. Las preguntas buscaron propiciar el intercambio de opiniones en torno a las temáticas bajo estudio (i.e. ¿Qué es ser buen ciudadano en Arica? ¿Qué dificultades existen para participar? ¿Qué aspectos controversiales de la Independencia Nacional consideran relevantes para sus estudiantes?). La entrevista fue registrada con grabadora de audio y transcrita en su totalidad. Las opiniones vertidas en este focus group fueron profundizadas en la segunda entrevista con la participante principal.

\section{Análisis y reporte de los datos}

Para el análisis de los datos se avanzó cíclicamente - a través de un proceso de reducción y reorganización de los datos - (Saldaña, 2013), y secuencialmente -por medio de la integración progresiva de datos provenientes de los distintos instrumentos de recolección. De este modo, se analizaron los datos separadamente partiendo por las entrevistas individuales. En un primer ciclo, se realizó una codificación con códigos deductivos provenientes del marco conceptual (ie. enseñanza de la Historia, temas socialmente relevantes, formación ciudadana, etc.), e inductivos tal como fueron emergiendo de los datos (i.e. temas contingentes, dificultades en el aula, realidad de los estudiantes). En un segundo ciclo, se 
integraron códigos afines bajo categorías aglutinadoras (ie. fines de la enseñanza de la Historia, enseñanza de la Independencia Nacional, temas sociales relevantes e Independencia Nacional, etc.). Posteriormente, se reiteraron estos ciclos, primero, con los datos provenientes de la entrevista grupal, y luego, con aquellos de las observaciones de clases. Los códigos utilizados se homologaron con aquellos empleados para analizar la entrevista individual. Este proceso, a su vez, sirvió para la triangulación de los datos verificando la consistencia interna entre ellos a pesar de provenir de fuentes distintas, y reforzando así su credibilidad (Mertens, 2015).

Finalmente, tras el análisis, se elaboró el reporte de los hallazgos en donde se integran el conjunto de datos recogidos. El reporte se construyó a partir de tres temas, que articulan las múltiples categorizaciones resultantes. Los dos primeros temas se refieren a las dos grandes temáticas de esta investigación: la enseñanza de la Historia y de la Independencia Nacional; y la enseñanza de la Independencia Nacional y la formación ciudadana de los estudiantes. Estos temas integran los datos provenientes de las entrevistas individuales que son complementados, para su profundización, por aquellos provenientes de la entrevista grupal. En cuanto al tercer tema, este agrupa aquellas categorías referidas a la práctica de enseñanza de la profesora, que derivan de las entrevistas individuales y las observaciones de clases, y que están directamente vinculadas a la investigación. Por último, la vinculación explícita entre los hallazgos, el objetivo de investigación y el marco conceptual se presenta más adelante en la discusión sobre los hallazgos.

\section{Hallazgos}

La enseñanza de la Historia y de la Independencia Nacional

Para la profesora Sonia, la enseñanza de la Historia suponía la centralidad del estudiante quien "debe generar su aprendizaje a través de la experiencia. La Historia nos da mucho de eso, porque todo lo que ha pasado se refleja en nuestro presente". De ahí, que la profesora también haya señalado que: "no me gusta esto de la memoria repetitiva, de aprenderse un dato solo por aprenderse un dato, no creo en una historia lineal". Por tanto, respecto de los contenidos del currículo, se oponía a entregar una versión definitiva de los acontecimientos históricos: "igual estoy enseñando lo que un niño de aquí hasta Punta Arenas tiene que aprender, pero con un valor agregado que es la opción de dialogar un poco más en clase, pensar y reflexionar". Esto fue complementado por otro profesor en la reflexión grupal: "yo personalmente uso un cuarenta por ciento el texto de estudio en mis clases. Lo otro, voy tomando decisiones y más que nada haciendo material y promoviendo otro tipo de discusiones, y no tanto las actividades del texto". Como resultado, los profesores participantes coincidían en que se debían introducir nuevos énfasis más allá de enseñar sólo fechas y nombres de personajes. Así lo ilustró otro profesor: "este país se cansó de los héroes, ahora estamos mucho más concentrados en los procesos, en entender las cuestiones colectivas". 
Sin embargo, la profesora Sonia también planteó dificultades para implementar nuevos modos de enseñar la Historia: "nuestra educación en Chile ha sido mucho de 'abran la página 74 y hagan la actividad 3' y esas actividades no invitan a algo más profundo". Desde su perspectiva, los contenidos deberían "ser cosas que [los estudiantes] sientan que los representan, [que] se aterrizan en su contexto". Si bien agradecía que en su escuela se diera "bastante libertad para el desarrollo de nuestras clases"; Sonia también sentía que, con sus colegas del Departamento de Historia, "conversamos más o menos las estrategias que tenemos, pero se comparte poco (...) Por ejemplo, una de las colegas usa harta guía y yo no lo hago (...) hacer como abuso de ese recurso para mí no es enriquecedor". En la conversación con otros participantes este aspecto fue refrendado por un profesor: "en el texto hay demasiados recursos que, a mi juicio, son muy monótonos, muy reiterativos, como el uso de las lecturas (...) si ponen datos visuales, gráficos, cuadros sinópticos, desemboca siempre en lo mismo". Asimismo, otro profesor planteó la dificultad para introducir cambios: "uno se tiene que alinear con este currículo rígido, (...) no puede salirse de eso para crear otras estrategias curriculares que sean mucho más motivantes para los propios estudiantes".

Consecuentemente, la profesora no estaba satisfecha con la propuesta curricular para enseñar la Independencia: "el programa me dice que la pase con líneas de tiempo y haga paralelos de Europa y América, pero se queda en eso, en el dato concreto, no hay desarrollo de pensamiento crítico". Por el contrario, era de la opinión que se podía alcanzar "un pensamiento más profundo" si se usaban distintas estrategias didácticas, tales como, separar a los estudiantes en grupos para que representaran los diferentes bandos que se enfrentaron en la Independencia y "defendieran su postura". Estos comentarios también fueron secundados por los otros profesores, como lo expresó uno: "Yo estoy convencido de que existen otras metodologías, los paneles, los foros, son una estrategia para la Independencia para provocar el debate". Respecto del valor de estudiar la Independencia para la formación del pensamiento histórico, un profesor lo describió señalando que a veces es difícil comprender que no siempre hubo repúblicas en el pasado: "hacer que los niños entiendan eso es complejo (...) la República no nació rápidamente, esto fue un proceso que se demoró". Del mismo modo, otra profesora concluyó que estudiar la Independencia permite a los estudiantes desarrollar "habilidades que lleven a razonar, pensar lógicamente, críticamente". Ello, a su vez, les permite valorar las instituciones que resultaron de la Independencia, "porque nos dan una organización. Pueden ser mejores, tienen que ser mejores, y esa mejoría va en la medida que los estudiantes se empoderen y desarrollen esas habilidades".

\section{Enseñanza de la Independencia y formación ciudadana}

La profesora Sonia también resaltó la importancia de vincular su enseñanza con la realidad de los estudiantes: "tenemos una generación muy controlada por las redes sociales y, de repente, aterrizarlos un poco y contextualizarlos, mostrarle noticias, es muy importante para que cambien el foco". De ahí que, procurara partir "las clases con temas contingentes (...) ahí ellos opinan. Hablamos de temas como el aborto, o las relaciones homosexuales". Como lo resumió: "uno los invita a subirse a este tren de la contingencia (...) y les dice 'mira, 
esto está pasando ahora, ¿qué hubieses hecho tú frente a eso? ¿qué opinas tú?'”. Desde la perspectiva de otro profesor, "nuestra misión como profesores de Historia es sensibilizarlos (...) si uno invita [a los estudiantes], son capaces de formular varios cambios en relación a estos temas y después ir discutiendo". Asimismo, los profesores plantearon la pertinencia de tratar temas actuales como la influencia del modelo económico imperante en el centro del país y que afectaba a la ciudad de Arica. Una profesora planteó que, si bien los estudiantes no critican el nuevo mall construido en la ciudad, siguiendo el modelo comercial instalado en otras partes del país, eso no significaba que "no se den cuenta que somos una región postergada. Lo señalan en sus cuestionamientos: 'esto no es el Estado que se acercó a nosotros y nos puso un mall', saben que hay una multinacional que tiene plata”.

Lo anterior entroncaba con el énfasis en formación ciudadana que la profesora Sonia introducía a su enseñanza. Ella lo resumió de la siguiente manera: "si seguimos haciendo una historiografía que no está hecha desde la ciudadanía, desde la comunidad, estamos repitiendo un patrón. El hacer historia va más allá (...). Generar una consciencia, saber quiénes somos, qué hacemos acá y qué podemos hacer". Para ello, buscaba conversar con sus estudiantes sobre "los derechos del niño, de la niña, de jóvenes, la búsqueda del bien común, el tema político, las leyes que se han creado (...) esos temas les gusta”. Entre los profesores también hubo consenso al respecto. Un profesor compartió su interés en que los estudiantes "conozcan ciertos parámetros y conceptos derivados de la Educación Cívica que son fundamentales como los deberes y derechos ciudadanos, el respeto por los derechos esenciales en relación a la construcción de la democracia". Haciendo eco de este parecer, una profesora lo resumió señalando que debiera buscarse que el estudiante "sienta propio los problemas de los Ariqueños y que se sitúe como parte de una solución (...), o sea, esto me afecta, pero yo también aporto. Si la reflexión en Historia no sirve para eso, entonces ¿para qué sirve?"

Desde una perspectiva ciudadana, la profesora Sonia estaba convencida que la enseñanza de la Independencia contribuía a la reflexión sobre diversos valores como la igualdad o la paz, pero de manera particular "la Independencia genera un aprendizaje en la actualidad para valorar la libertad”. Asimismo, en la conversación entre los profesores, sobresalió la temática de la exclusión en relación a la Independencia. Así, una profesora planteó que la Independencia "es una historia de hombres y que son héroes, porque esos son los que protagonizan nuestra lectura oficial de la Independencia y además es la historia del centro [del país]. Las regiones, nosotros, no tenemos participación ahí". Aquello fue complementado por la crítica de un profesor: "El currículo de Historia está desprovisto de la cuestión regionalista y eso desvirtúa el concepto que los estudiantes manejan sobre la Independencia. Es necesario que el alumno se vea involucrado en la construcción democrática de su región".

\section{Reflexionando en el aula sobre los valores de la Independencia}

Específicamente, en relación a la clase sobre la Independencia Nacional que observamos enseñar a la profesora Sonia, hubo muchos aspectos coincidentes con lo planteado en las entrevistas. Si bien, se ciñó a la estructura planteada en el texto escolar sobre los temas de la Ilustración y la Revolución Francesa, mantuvo una constante interacción con los 
estudiantes y procuró que vincularan los contenidos con situaciones de la actualidad a través de preguntas y comentarios. Así, haciendo mención sobre los sentimientos de las colonias americanas y la influencia que sobre ellas tuvo el "lema de la libertad, igualdad y fraternidad", preguntó: "Hoy en día, en el siglo XXI ¿tienen la oportunidad los chilenos de no estar controlados económicamente?" Tras una ronda de respuestas breves por parte de los estudiantes, concluyó: "Si tú te pones a comparar la dinámica que teníamos en la Colonia con la dinámica que tenemos en la actualidad nos vemos en la misma situación, porque los recursos naturales son administrados por empresas extranjeras". También organizó algunas actividades grupales, con plenarios finales, en donde algunos estudiantes pudieron explayar sus respuestas, tal como lo hizo una alumna al hablar de la autonomía alcanzada: "Chile no es totalmente independiente porque hay algunos países que tienen mucha influencia, tanto en la economía como en la sociedad. Chile negocia con ellos y está lleno de productos que son de allá".

La profesora Sonia quedó conforme con sus clases sobre la Independencia, aunque estaba consciente que los estudiantes no habían participado tanto como hubiese deseado. Desde su perspectiva, entre los estudiantes "hay una indiferencia o desgano con esto de estar más actualizado con la información y su manejo, cuestionar un poco más". Ello explicaba que, en sus clases, "no haya pasado que exista un debate de horas y horas (...) y, finalmente, se genere un diálogo. Ellos carecen de argumentos y entonces llega hasta cierto límite sus opiniones". Sin embargo, quedó conforme con el rendimiento de los estudiantes en la unidad, y, en particular, con las respuestas que, dentro de una evaluación, dieron a una pregunta abierta sobre la Ilustración y la centralidad del pensamiento para promover cambios sociales. Como lo describió, los estudiantes "respondían cosas como ¡tú puedes hacerlo!, ¡sí se puede!, hay que pensar y es importante razonar". También los alumnos señalaron su conformidad con haber aprendido sobre la Independencia, lo que la profesora Sonia atribuyó a que lo enseñado "se relacionó con el día a día de ellos; porque llevarlo a un contexto de cientos de años, es ya muy lejano y tiene poco interés". Como prueba de ello, una estudiante, al finalizar la segunda clase observada, valoró el haber aprendido sobre "el origen de la Independencia (...) ahora la gente vive en malas situaciones, pero antes era mucho peor, porque vivían con miedo. Ahora la gente vive con rabia o con pena, pero no con miedo". A ello, la profesora Sonia replicó concluyendo: "Confío que hayan quedado con la sensación que deben defender la igualdad, la libertad y deben ser fraternos entre ustedes para buscar el bien común, y eso es ser cada vez más libres, vivir en una sociedad más justa”.

\section{DISCUSIÓN}

A partir de los hallazgos y teniendo en cuenta el marco conceptual, ahora discutimos acerca del uso que la profesora hace de una metodología de enseñanza no lineal de la Historia que le permitió establecer vinculaciones con la realidad de sus estudiantes; la que incluyó establecer relaciones entre la temática de la Independencia Nacional y temáticas socialmente relevantes que aportan a la formación ciudadana de estos estudiantes. También sugerimos algunas líneas de investigación que profundicen las conclusiones preliminares de este estudio en el futuro. 


\section{La enseñanza de la Historia y su vinculación con los estudiantes}

La profesora de nuestro estudio visualiza el alcance, en la escuela, de los diversos sentidos y contenidos que han ido conformando los diferentes modelos de enseñar la Historia, tal como los describimos en el marco conceptual (Rosa \& Brescó, 2017). En ese sentido, es ilustrativo como la profesora se plantea en contra de una forma de enseñanza que enfatiza meramente la transmisión de lecturas sobre el pasado basadas en 'una historia lineal'. Esta opción es compartida por los demás profesores que participaron de esta investigación. Consecuentemente, en vez de presentar una versión de la historia descriptiva a través de hechos y/o personajes, la profesora adhiere a una concepción de la enseñanza de la Historia que, en líneas generales, coincide con aquella cuyos propósitos son la reflexión y el pensamiento crítico (Seixas, 2017). Sin embargo, su opción metodológica no pareció descansar tanto en un interés disciplinario como en establecer vinculaciones con sus estudiantes tratando de concederles más participación e intentando hacer pertinentes los contenidos de enseñanza a sus contextos culturales o experiencias vividas. Desde esa perspectiva, la experiencia de la profesora Sonia sugiere que, formas más reflexivas de enseñar la Historia no sólo cumplen con finalidades propiamente historiográficas, sino también relacionales favoreciendo el diálogo y la interacción en el aula.

No obstante, de estos hallazgos también se desprenden aspectos problemáticos. Por un lado, la profesora Sonia, junto a los demás profesores, se muestra crítica con los contenidos y metodologías de enseñanza que se proponen en el currículo prescrito y en la escuela. Si bien no era objetivo de esta investigación ahondar en la comprensión de este tipo de aspectos contextuales, subrayamos su importancia al momento de pensar cómo los profesores podrían vincular mejor los contenidos de la Historia con las experiencias de los estudiantes. Por otro lado, en lo expuesto por los docentes, tampoco percibimos mayor reflexión sobre cómo deber ser mediatizados los contenidos para que los estudiantes adquieran un aprendizaje más profundo sobre la Historia, que les ayude a ampliar su actual comprensión de la realidad. Un ejemplo, observado en la clase de la profesora Sonia, es su afirmación de que, tanto en la Independencia Nacional como hoy, "nos vemos en la misma situación", lo que no se condice con principios de continuidad y cambio en la historia (Lee \& Shemilt, 2011). Lo anterior deja interrogantes respecto del alcance, más bien genérico, de la vinculación que ella establece entre los contenidos de Historia y el aprendizaje de los estudiantes, y que no les contribuye suficientemente con los instrumentos teóricos para que aborden los relatos del pasado, estableciendo relaciones con el presente y proyectando hacia el futuro su reflexión acerca de la sociedad (Carpente \& López Facal, 2013).

\section{La enseñanza de la Independencia Nacional y los temas socialmente relevantes}

Habiendo reconocido, a pesar de sus limitaciones, el valor de una metodología de enseñanza no lineal de la Historia para que la profesora Sonia pudiera establecer vínculos con los estudiantes, también valoramos que la profesora se haya valido de esa vinculación para intentar establecer conexiones entre los contenidos de enseñanza y problemáticas 
contingentes referidas a la ciudadanía. Ello es igualmente congruente con algunos supuestos establecidos en el marco conceptual conforme a los cuales el desarrollar habilidades de pensamiento crítico y argumentación en el aula suponen integrar perspectivas que derivan de temas públicos socialmente relevantes (De Amézola, 2008). En consonancia con ello, en el decir de la profesora y, compartido por los demás profesores participantes, el estudio de la Independencia Nacional efectivamente tiene implicancias para entender los conflictos de la sociedad actual, lo que precisamente contribuye a reflexionar sobre la relación pasado, presente y futuro, promoviendo que los estudiantes se sientan parte de los problemas en su contexto y se sitúen como parte de la solución. En ese sentido, es de importancia que estos profesores procuraran conversar con sus estudiantes sobre temáticas ciudadanas particulares de su región, enmarcándolas bajo dinámicas nacionales y globales más amplias. En ese contexto, la Independencia Nacional, al ser comprendida, al mismo tiempo, como un proceso nacional e internacional, constituye un contenido de enseñanza de la Historia que permite poner en perspectiva temporal y geográfica los problemas que afectan actualmente a lo local y que están asociados a la pervivencia de valores ciudadanos fundamentales como la libertad o la participación.

Consiguientemente, constituyen un aporte los intercambios profesora-alumnos que pudimos observar en las clases de la profesora Sonia, gracias a su estilo interactivo de enseñanza, ya que posibilitaron la formulación de temáticas, tales como la dependencia económica o la desigualdad social, cuya comprensión requiere de contenidos históricos más distantes en el tiempo, como la Independencia Nacional. Desde esa perspectiva, estos hallazgos parecen reafirmar, en primer lugar, que no sólo determinados contenidos históricos más actuales, sino también aquellos más lejanos en el tiempo son pertinentes a la formación ciudadana. En segundo lugar, que ello debe ir acompañado de una interacción constante entre el profesor y sus estudiantes, lo que, de acuerdo a los profesores de este estudio, implica también incorporar estrategias didácticas específicas que ayuden a los estudiantes a reconocer la relación que existe entre los contenidos históricos y la responsabilidad que habrán de tener como futuros ciudadanos participativos para resolver los problemas de convivencia en una sociedad democrática (López Facal \& Santidrián, 2011). No obstante, a la luz de nuestras observaciones y a lo compartido por la profesora Sonia, debemos constatar que no observamos mayormente el uso de estrategias tales como el trabajo de fuentes, la deliberación sobre interpretaciones contrapuestas, la elaboración de narrativas a partir de las comprensiones alcanzadas, o la investigación de factores contextuales y causales de las temáticas bajo estudio. Pero las causas de ello, podrían descansan en las limitaciones ya mencionadas, presentes en el currículo y en la propia escuela.

\section{Contribuciones a los estudios en el área y preguntas de investigación a futuro}

El conjunto de perspectivas discutidas aporta con un referente empírico a las investigaciones que procuran establecer la vinculación que debiera darse entre la enseñanza de la Historia, los contenidos de enseñanza y temáticas relevantes para la formación ciudadana de los estudiantes. De manera particular, contribuye a comprender el alcance 
para la formación ciudadana de los contenidos referidos a la enseñanza de la Independencia Nacional, y últimamente, a la importancia de estudiar eventos más distantes en el tiempo, pero que igualmente aportan al desarrollo ciudadano de los estudiantes; una temática menos explorada en la literatura. De este modo, este estudio sugiere algunos temas más específicos que podrían tratarse al momento de enseñar este tipo de contenido histórico, aunque al mismo tiempo advierte, a la luz de sus hallazgos, que estos temas finalmente vendrán dados por la diversidad cultural y social de los estudiantes, y los contextos locales en los que se desenvuelven. Ante ello, la interrelación que los profesores establezcan con sus estudiantes será fundamental, aunque ciertamente dicha interrelación se verá favorecida por un modelo de enseñanza de la Historia que procure mover a la reflexión crítica y no a la mera repetición de narrativas oficiales (Pagès, 2016).

Asimismo, este estudio aporta una serie de aspectos que deben ser profundizados a través de investigaciones a futuro dada la aproximación exploratoria utilizada. En primer lugar, se requieren estudios centrados en estudiantes, con muestras más representativas del carácter global que adquiere la ciudadanía, para reconocer mejor aquellas temáticas ciudadanas que son relevantes para la enseñanza de eventos distantes en el tiempo, y viceversa. Ello permitirá verificar también algunas afirmaciones de la profesora Sonia acerca de las limitaciones de sus estudiantes para involucrarse en procesos críticos reflexivos más profundos, y vislumbrar las acciones pedagógicas correspondientes, más allá de las particularidades de grupos determinados de estudiantes. En segundo lugar, se hace necesario conformar diseños investigativos más sofisticados que logren capturar la complejidad -no siempre reconocida- que importa vincular contenidos del pasado con aquellos del presente, y que además sean relevantes para que los estudiantes piensen en su futuro como ciudadanos. Según se deduce de este estudio, todo lo anterior supone procesos metodológicos que muchas veces deben articularse en contextos curriculares y escolares adversos que incluso tienden a la simplificación. Desde esa perspectiva, futuros estudios deberán atender a estas circunstancias junto con proponer propuestas metodológicas que integren más balanceadamente y exhaustivamente los objetivos de la enseñanza de la Historia con aquellos provenientes del campo de la Formación Ciudadana.

\section{CONCLUSIONES}

El objetivo de este trabajo era analizar, desde la perspectiva de una profesora de la ciudad de Arica, la relación que establece entre la enseñanza de la Historia, los contenidos sobre la Independencia Nacional y temáticas relevantes para la formación ciudadana de los estudiantes. Los hallazgos nos permiten concluir que una metodología de enseñanza no lineal de la Historia ayudó a la profesora a establecer vinculaciones con sus estudiantes más allá de lo disciplinario, aunque, por un lado, los profesores participantes señalan una serie de obstáculos presentes en el currículo y sus escuelas que dificultan esta tarea y, por otro, parecieran dejar en un plano más bien secundario la promoción de habilidades como el pensamiento histórico. Junto a lo anterior, la vinculación que la profesora establece con sus estudiantes gracias a su metodología de enseñanza de la Historia, le sirvió, además, 
para vincular los contenidos de la Independencia Nacional con temáticas que son relevantes socialmente -como la dependencia económica o las tensiones entre la región de Arica y el centro del país- y que ayudan a preparar para una ciudadanía conectada con las experiencias de los estudiantes. A la luz de ello, se reafirma, en primer lugar, que contenidos históricos más lejanos en el tiempo también son pertinentes a la formación ciudadana y, en segundo lugar, que requieren ser apoyados por estrategias didácticas más específicas para que efectivamente sean relevantes para los estudiantes. No obstante, nuestras observaciones dan cuenta de la complejidad de estos aspectos, lo que abre preguntas de investigación que en el futuro debieran buscar establecer de manera más precisa cómo se debieran interrelacionar más profundamente objetivos y contenidos que vienen de ámbitos distintos, como lo son la enseñanza de la Historia y la Formación Ciudadana.

Últimamente, este estudio también reafirma que los profesores son actores claves en una enseñanza de la Historia orientada a formar ciudadanos. Asimismo, ilustra cómo los docentes, a través de sus decisiones, posibilitan que sus estudiantes analicen críticamente el pasado, y puedan debatir sobre cuestiones públicas del presente y desde ahí puedan proyectar el futuro; cuestión fundamental para la construcción de una sociedad democrática. Al mismo tiempo, invita a continuar estudiando cómo los profesores, tanto individual como colectivamente, pueden mejorar las decisiones que toman respecto de qué enseñar y qué aprendizajes promover, además de reflexionar sobre cómo una enseñanza significativa de la Historia puede contribuir a la formación de la ciudadanía, y viceversa. Sin embargo, en ello, este trabajo igualmente advierte respecto de los condicionamientos contextuales que provienen tanto del currículo como de la escuela, y que dificultan este tipo de enseñanza. Investigaciones a futuro debieran considerar con mayor centralidad este aspecto, para no solo apuntar a las falencias de los profesores al momento de enseñar, sino también para sugerir las estrategias que les ayuden a sortear los obstáculos presentes. Tanto la Historia como la conciencia ciudadana tienen mucho que aportar al respecto.

\section{REFERENCIAS}

Arthur, J., Davies, I., Wrenn, A., Haydn, T., \& Kerr, D. (2001). Citizenship through secondary history. London: Routledge.

Barton, K., \& McCully, A. (2007). Teaching controversial issues...where controversial issues really matter. Teaching History, 127, 13-19.

Barton, K., \& Levstik, L. (2009). Teaching for the Common Good. New York: Routledge.

Carpente, L., \& López Facal, R. (2013). Argumentación y competencias en la enseñanza de las ciencias sociales. Íber Didáctica de las Ciencias Sociales, Geografía e Historia, 74, 43-51.

Carretero, M. (2007). Documentos de Identidad. La construcción de la memoria histórica en un mundo global. Buenos Aires: Paidós.

Carretero, M; Asensio M., \& Rodríguez-Moneo, M. (Eds.) (2012). History Education and the Construction of National Identities. Charlotte: IAP.

Conrad, J. (2020). Navigating identity as a controversial issue: One teacher's disclosure for critical empathic reasoning. Theory y Research in Social Education, 48(2), 211- 243. 
De Amézola, G. (2008). Currículo oficial y memoria. El pasado reciente en la escuela argentina. Enseñanza de las ciencias sociales, 7, 47-55.

Goldberg, T., \& Savenije, G. M. (2018) Teaching Controversial Historical Issues. En S. Metzger, \& L. Harris (Eds.), The Wiley international handbook of history teaching and learning (pp. 503-526). New York: Wiley Blackwell. http://doi.org/10.1002/9781119100812.ch19

Haste, H., \& Bermudez, A. (2017). The power of story: Historical narratives and the construction of civic identity. En M. Carretero, S. Berger y M. Grever (Eds.), Palgrave handbook of research in historical culture and education (pp. 427-448). London: Palgrave Macmillan.

Hess, D. (2011). Discussions that drive democracy. Educational Leadership, 69(1), 69-73.

Hess, D., \& McAvoy, P. (2015). The Political classroom. Evidence and ethics in democratic education. New York: Routledge.

Kello, K. (2016). Sensitive and controversial issues in the classroom: teaching history in a divided society. Teachers and Teaching: theory and practice, 22(1), 35-53.

Landa, L., \& Pinto, V. (2017). Historia, geografía y ciencias sociales. $8^{\circ}$ Básico. Texto del estudiante. Santiago de Chile: Editorial SM.

Lee, P., \& Shemilt, D. (2011). The concept that dares not speak its name: Should empathy come out of the closet? Teaching History, 143, 39- 49.

López Facal, R., \& Santidrián, V. (2011). Los «conflictos sociales candentes» en el aula. Íber: Didáctica de las Ciencias Sociales, Geografía e Historia, 69, 8-20.

Magendzo, A. (2016). Incorporando la perspectiva controversial en el currículum disciplinario. Revista Iberoamericana de Educación Superior, VII(19),118-130.

Martínez-Rodríguez, R.; Muñoz, C., \& Sánchez-Agustí, M. (2019). Conocimientos y creencias del profesorado y su vínculo con las finalidades de la enseñanza de la historia reciente. La transición a la democracia en España como caso controvertido. Revista de Educación, 383, 11-35.

McAvoy, P., \& Hess, D. (2013). Classroom deliberation in an era of political polarization. Curriculum Inquiry, 43(1), 14-47.

Mertens, D. M. (2015). Research and evaluation in education and psychology: Integrating diversity with quantitative, qualitative, and mixed methods. California: Sage publications.

Metzger, S., \& Harris, L. (Eds.) (2018). The Wiley international handbook of history teaching and learning. New York: Wiley Blackwell.

Miralles, P., Prats, J., \& Tatjer, M. (2012). Conocimientos y concepciones de estudiantes españoles y latinoamericanos de educación secundaria obligatoria sobre las independencias políticas americanas. Scripta Nova Revista Electrónica de Geografía y Ciencias Sociales, 418(34), 1-8.

Montanares-Vargas, E. (2017). Enseñanza de la historia en la Araucanía, Chile: un estudio de las representaciones sociales del profesorado en formación. Prisma social, 19, 1-18.

Neuman, L. (2014). Social research methods: Qualitative and quantitative approaches. England: Pearson Education Limited.

Niens, U., Connor, U., \& Smith, A. (2013). Citizenship education in divided societies: teachers' perspectives in Northern Ireland. Citizenship Studies, 17(1), 128-141.

Nordgren, K., \& Johansson, M. (2015). Intercultural historical learning: a conceptual framework, 
Journal of Curriculum Studies, 47(1), 1-25

Ocampo, L., \& Valencia, S. (2019). Los problemas sociales relevantes: enfoque interdisciplinar para la enseñanza integrada de las ciencias sociales. Revista de Investigación en Didáctica de las Ciencias Sociales, 4, 60-75.

Pagès, J. (2016). Enseñar ciencias sociales, geografía e historia desde la perspectiva de la ciudadanía democrática. Cuaderno de Educación, 72, 1-9.

Pagès, J. (2019). Enseñar historia, educar la temporalidad, formar para el futuro. El Futuro del Pasado, 10, pp. 19-56.

Pagès, J, Villalón, G., \& Zamorano, A. (2017) Enseñanza de la Historia y Diversidad Étnica: los casos chileno y español. Educação y Realidade, 42(1), 161-182.

Pineda, J. (2015). Educar para la ciudadanía trabajando con temas controvertidos en Educación Secundaria Obligatoria. Revista de Investigación Educativa, 33(2), 353-367.

Pinto, J., \& Valdivia, V. (2009). ¿Chilenos todos? La construcción social de la nación (18101840). Santiago de Chile: LOM ediciones.

Rosa, A., \& Brescó, I. (2017). What to teach in history education when the social pact shakes? En M. Carretero, S. Berger y M. Grever (Eds.), Palgrave handbook of research in historical culture and education (pp. 413-425). London: Palgrave Macmillan.

Saldaña, J. (2013). The coding manual for qualitative researchers. California: SAGE.

Sáiz, J., \& López Facal, R. (2012). Aprender y argumentar España. La visión de la identidad española entre el alumnado al finalizar el bachillerato. Didáctica de las ciencias experimentales y sociales, 26, 95-120.

Salinas, J., \& Oller, M. (2017). Debatiendo temas controversiales para formar ciudadanos. Una experiencia con alumnos de secundaria. Praxis educativa, XXI(3), 40-48.

Santisteban, A. (2019). La enseñanza de las Ciencias Sociales a partir de problemas sociales o temas controvertidos: estado de la cuestión y resultados de una investigación. El Futuro del Pasado, 10, 57-79.

Seixas, P. (2017). Historical consciousness and historical thinking. En M. Carretero, S. Berger y M. Grever (Eds.), Palgrave handbook of research in historical culture and education (pp. 59-72). London: Palgrave Macmillan.

Schuitema, J., Radstake, H., van de Pol, J., \& Veugelers, W. (2018). Guiding classroom discussions for democratic citizenship education. Educational Studies, 44(4), 377-407.

Swalwell, K., \& Schweber, S. (2016). Teaching through turmoil: social studies teachers and local controversial current events. Theory \& Research in Social Education, 44, 283-315.

Toledo, M., Magendzo, A., Gutiérrez, V., \& Iglesias, R. (2015). Enseñanza de 'temas controversiales' en la asignatura de historia y ciencias sociales desde la perspectiva de los profesores. Estudios Pedagógicos, XLI(1), 275-292.

Van Boxtel, C., \& van Drie, J. (2018). Historical Reasoning: Conceptualizations and Educational Applications. En S. Metzger y L. Harris (Eds.), The Wiley international handbook of history teaching and learning (p. 149-176). New York: Wiley Blackwell.

Vásquez, G. Sánchez, M. Vásquez, N., \& Muñoz, C. (2018). La transición democrática chilena ¿proceso pactado o brusca ruptura?: una mirada desde la perspectiva de los estudiantes secundarios. Tempo e Argumento, 10(24), 247 - 278. 


\section{G. Villalón-Gálvez et al.}

Zembylas, M., \& Kambani, F. (2012). The teaching of controversial issues during elementarylevel history instruction: Greek-Cypriot teachers' perceptions and emotions. Theory $y$ Research in Social Education, 40, 107-133. 